Frühe Progesteron-Gabe bei akutem SHT

\section{Kein klinischer Effekt}

Fragestellung: Ist eine frühe Gabe von Progesteron bei der Behandlung des akuten Schädel-Hirn-Traumas (SHT) wirksam?

Hintergrund: In den USA werden pro Jahr 2,4 Millionen Menschen in Emergency Rooms wegen SHT behandelt. Progesteron ist ein potentes Neurosteroid, das in Tierexperimenten bei SHT zu einer Reduktion des Hirnödems und neurologischer Ausfälle führt. Zwei kleine prospektive Studien hatten bei Patienten mit SHT positive Ergebnisse einer frühen Behandlung mit Progesteron ergeben $[1,2]$.

Patienten und Methodik: In die Doppelblindstudie wurden Patienten mit SHT mit einem Score auf der Glasgow Coma Scale (GCS) zwischen 4 und 12 eingeschlossen. Die Behandlung begann innerhalb von vier Stunden, die Patienten erhielten entweder 0,05 mg Progesteron pro kg Körpergewicht oder Placebo. Die Therapie erstreckte sich über 96 Stunden. Der primäre Endpunkt war der Outcome nach sechs Monaten auf der extended GCS.

Ergebnisse: Für die Studie wurden 17.681 Patienten gescreent, 882 Patienten wurden randomisiert. Die Studie wurde vorzeitig wegen „futility“ abgebrochen. Die Patienten waren im Mittel 35 Jahre alt, drei Viertel waren Männer. Die häufigste Ursache für das SHT waren Verkehrsunfälle. Im Mittel vergingen $163 \mathrm{Mi}$ nuten zwischen Eintreffen in der Notaufnahme und Randomisierung. Für keinen der präspezifizierten Endpunkte ergab sich eine Überlegenheit von Progesteron gegenüber Placebo.
Wright DW, Yeatts SD, Silbergleit R et al; NETT Investigators. Very early administration of progesterone for acute traumatic brain injury. N Engl J Med 2014; $371: 2457-66$
Schlussfolgerungen: Diese große Studie aus den USA zeigte keinen klinischen Effekt einer frühen Behandlung mit Progesteron bei $\mathrm{Pa}$ tienten mit mittelschweren und schweren SHT.

\section{- Kommentar von Hans-Christoph Diener, Essen}

\section{Fortsetzung der Negativreihe}

Die Behandlung des SHT bewegt sich offenbar in dieselbe Richtung wie die neuroprotektive Therapie beim akuten ischämischen Insult. Bisher sind alle Studien zur medikamentösen Therapie dieser Erkrankung negativ verlaufen. Dies gilt auch für die hier durchgeführte Studie mit dem frühen Einsatz von Progesteron bei Patienten mit akuten SHT. Die Studie zeigt auch, dass sich offenbar Ergebnisse aus Tierexperimenten und kleinen Phase-Il-Studien nicht auf große Populationen übertragen lassen.

\footnotetext{
Referenzen:

1. Wright DW et al. Ann Emergency Med 2007; 49: 391 - 402

2. Xiao G et al. Critical Care 2008; 12: R61
}

\section{Keine Neuroprotektion}

Fragestellung: Ist Progesteron bei Patienten mit schweren Schädel-Hirn-Traumen (SHT) wirksam?

Hintergrund: Beim SHT kommt es zu einer Vielzahl pathophysiologischer Vorgänge wie Hirnödem, axonalen Schäden und Blutungen sowie zerebralen Ischämien. Progesteron hat in her schien es gerechtfertigt, eine größere Studie an Patienten durchzuführen.

Patienten und Methodik: Es handelte sich um eine multinationale placebokontrollierte Studie, in die 1.195 Patienten im Alter zwischen 16 und 70 Jahren mit schweren SHT eingeschlossen wurden. Einschlusskriterien war ein Wert auf der Glasgow Coma Scale $(\mathrm{GCS}) \leq 8$. Die Behandlung begann innerhalb von acht Stunden nach der Verletzung und wurde für 120 Stunden durchgeführt. Die initiale Dosis betrug Progesteron 0,71 mg pro kg Körpergewicht pro Stunde und wurde dann nach einer Stunde mit 0,50 mg pro kg Körpergewicht über 119 Stunden durchgeführt. Der primäre Endpunkt war der GCS-Wert nach sechs Monaten.

Ergebnisse: Die Patienten waren im Mittel 35 Jahre alt und fast $80 \%$ waren Männer. Bei den meisten SHT handelte es sich um Verkehrsunfälle. Etwa $10 \%$ der Patienten hatten einen GCSWert von 3, $45 \%$ zwischen 4 und 6 und $44 \%$ zwischen 7 und 8 . Für den primären und alle sekundären Endpunkte ergaben sich keine Unterschiede zwischen Progesteron und Placebo. Dies galt auch für die Sterblichkeit oder den Verbleib im Koma, was für beide Gruppen $22 \%$ betrug, $32 \%$ hatten einen guten Out-

Skolnick BE, Maas Al, Narayan RK et al; SYNAPSE Trial Investigators. A clinical trial of progesterone for severe traumatic brain injury. N Engl J Med 2014; 371: $2467-76$ come.

Schlussfolgerungen: Eine Behandlung mit Progesteron ist beim schweren SHT nicht wirksam. Tierexperimenten neuroprotektive Eigenschaften gezeigt. Da-

\section{- Kommentar von Hans-Christoph Diener, Essen Wirksame Therapie fehlt weiterhin}

Diese große internationale Studie mit Schwerpunkt in Europa zeigt ebenso wie die ebenfalls hier referierte Studie aus den USA keinen Nutzen einer Behandlung mit Progesteron beim SHT. Nimmt man diese beiden großen Studien zusammen, besteht weder eine Wirksamkeit bei sehr früher Behandlung (amerikanische Studie), noch eine Wirksamkeit bei schweren SHT (europäische Studie). Daher steht eine wirksame neuroprotektive Therapie beim SHT weiterhin aus. 